\title{
Lie Group Analysis of a Forced KdV Equation
}

\author{
Motlatsi Molati $^{1,2}$ and Chaudry Masood Khalique ${ }^{1}$ \\ ${ }^{1}$ Department of Mathematical Sciences, International Institute for Symmetry Analysis and Mathematical Modelling, \\ North-West University, Mafikeng Campus, Private Bag X 2046, Mmabatho 2735, South Africa \\ ${ }^{2}$ Department of Mathematics and Computer Science, National University of Lesotho, P.O. Roma 180, Lesotho
}

Correspondence should be addressed to Motlatsi Molati; m.molati@gmail.com

Received 26 January 2013; Accepted 26 February 2013

Academic Editor: Fazal M. Mahomed

Copyright (C) 2013 M. Molati and C. M. Khalique. This is an open access article distributed under the Creative Commons Attribution License, which permits unrestricted use, distribution, and reproduction in any medium, provided the original work is properly cited.

The Korteweg-de Vries (KdV) equation considered in this work contains a forcing term and is referred to as forced KdV equation in the sequel. This equation has been investigated recently as a mathematical model for waves on shallow water surfaces under the influence of external forcing. We employ the Lie group analysis approach to specify the time-dependent forcing term.

\section{Introduction}

Many studies in mathematical physics, engineering, life sciences, and in all other sciences use mathematical models to describe certain phenomenon. Such models are represented by the nonlinear evolution equations. The existence of analytical solutions to these equations becomes a basis for a better understanding of the dynamics involved. The KdV equation [1] being a nonlinear evolution equation models the nonlinear wave phenomena on the shallow water surfaces, and its various forms have been proposed depending upon the applications in diverse fields of science and engineering. To date, a lot of solution procedures, both analytical and numerical, have been developed to solve these types of differential equations. However, some of these approaches may involve approximation of solutions. The current work is based upon the Lie group theory approach as a solution procedure.

The Lie point or higher-order symmetries of a differential equation enable one to obtain the solutions invariant under a particular symmetry or a linear combination of symmetries. The invariant solutions are a basis to finding exact solutions or numerical solutions. In most real-life applications, the differential equations, which are used to model a certain situation, contain arbitrary functions of dependent variable or its derivatives and independent variables. Instead of assuming the forms of these model parameters, the method of group classification can be employed to systematically specify their forms. There are various approaches to solving a group classification problem, namely, the direct analysis or the approach based upon the equivalence transformations.

We consider the forced KdV equation

$$
u_{t}+c u_{x}+\alpha u u_{x}+\beta u_{x x x}=F(t),
$$

where $u$ represents the elevation of free water surface and $\alpha$ and $\beta$ are arbitrary constants which depend upon the long wave speed, $c$. The arbitrary smooth function $F(t)$ is the forcing term. The variables $t$ and $x$ represent time and space, respectively. This equation was proposed in [2] and the Hirota's bilinear approach was used to obtain the multiple soliton solutions. However, it is worth mentioning that $F(t)$ remained unspecified. In [3] the generalized wave transformation was used to transform a forced $\mathrm{KdV}$ equation with time-dependent coefficients into a homogeneous equation, and the soliton solutions were obtained by making use of the solitary wave ansatz. Likewise, the time-dependent coefficients remained arbitrary. The symmetry-based approach is given in [4-6]; the investigations in these references include approximate symmetry classification, derivation of some conservation laws, and the construction of the solutions. As mentioned earlier, the arbitrary functions assume their forms in a systematic fashion via the method of group classification. This approach has been utilized on similar equations $[7,8]$ to the underlying equation. 
TABLE 1: Classification results.

\begin{tabular}{|c|c|c|c|}
\hline No. & $F$ & Condition on consts. & Extension of principal Lie algebra \\
\hline 1. & $F_{0}(n+t)^{-5 / 3}+\frac{P}{5 \alpha}$ & $\alpha, F_{0}, p \neq 0$ & $X_{3}=6 \alpha(n+t) \partial_{t}+\alpha\left(p t^{2}+2 x\right) \partial_{x}+2[p t-2(c+\alpha u)] \partial_{u}$ \\
\hline 2. & $F_{0}+\frac{q t}{3 \alpha}$ & $\alpha, q \neq 0$ & $X_{3}=6 \alpha \partial_{t}+\alpha q t^{2} \partial_{x}+2 q t \partial_{u}$ \\
\hline 3. & $F_{0}(n+t)^{-5 / 3}$ & $F_{0} \neq 0$ & $X_{3}=3 \alpha(n+t) \partial_{t}+\alpha x \partial_{x}-2(c+\alpha u) \partial_{u}$ \\
\hline 4. & $F_{0}$ & $F_{0} \neq 0$ & $\begin{array}{c}X_{3}=\partial_{t}, \\
X_{4}=6 \alpha \partial_{t}+\alpha\left(5 \alpha F_{0} t^{2}+2 x\right) \partial_{x}+2\left[5 \alpha F_{0} t-2(c+\alpha u)\right] \partial_{u}\end{array}$ \\
\hline
\end{tabular}

Here $F_{0}, n, p$ and $q$ are arbitrary constants.

This work is organized as follows. The next section deals with the generation of the determining equations for Lie point symmetries and includes the classifying relation for the forcing term. The functional forms of the forcing term are specified via the direct analysis of the classifying relation. In Section 3, some results of the Lie group analysis are utilized for symmetry reductions and exact solutions. Finally, we summarize our investigations in Section 4.

\section{Lie Group Analysis}

In Lie's algorithm (see [9-11] for more details), a vector field

$$
X=\xi^{1}(t, x, u) \partial_{t}+\xi^{2}(t, x, u) \partial_{x}+\eta(t, x, u) \partial_{u}
$$

is a generator of Lie point symmetries of (1) if and only if

$$
\left.X^{[3]}\left(u_{t}+c u_{x}+\alpha u u_{x}+\beta u_{x x x}-F(t)\right)\right|_{(1)}=0,
$$

where

$$
X^{[3]}=X+\zeta_{t} \partial_{u_{t}}+\zeta_{x} \partial_{u_{x}}+\zeta_{x x x} \partial_{u_{x x x}}
$$

is the third prolongation of the vector field $X$. The variables $\zeta$ 's are given by the formulae

$$
\begin{gathered}
\zeta_{t}=D_{t}(\eta)-u_{t} D_{t}\left(\xi^{1}\right)-u_{x} D_{t}\left(\xi^{2}\right), \\
\zeta_{x}=D_{x}(\eta)-u_{t} D_{x}\left(\xi^{1}\right)-u_{x} D_{x}\left(\xi^{2}\right), \\
\zeta_{x x x}=D_{x}\left(\zeta_{x x}\right)-u_{x x t} D_{x}\left(\xi^{1}\right)-u_{x x x} D_{x}\left(\xi^{2}\right),
\end{gathered}
$$

where

$$
D_{t}=\partial_{t}+u_{t} \partial_{u}+\cdots, \quad D_{x}=\partial_{x}+u_{x} \partial_{u}+\cdots
$$

are the total derivative operators [10].

The invariance conditions (3) are separated with respect to the powers of the derivatives of $u$, and this yields the determining equations, which are a system of linear partial differential equations of homogeneous type in $\xi^{1}, \xi^{2}$, and $\eta$. It is easy but tedious to generate the determining equations manually. Nowadays there are many computer software packages for symbolic computation, which have been developed to find symmetries interactively or automatically. However, we are yet to develop the software package which solves the group classification problem whether complete or partial group classification.
The coefficients of the generator of Lie point symmetries (2), namely, $\xi^{1}, \xi^{2}$, and $\eta$, satisfy the determining equations

$$
\begin{gathered}
\xi_{u}^{1}=0, \quad \xi_{u}^{2}=0, \quad \eta_{u u}=0, \quad \xi_{x}^{1}=0, \\
\eta_{x u}-\xi_{x x}^{2}=0, \quad 3 \xi_{x}^{2}-\xi_{t}^{1}=0, \\
\xi^{1} F^{\prime}(t)+\left(\xi_{t}^{1}-\eta_{u}\right) F(t)-(c+\alpha u) \eta_{x}-\beta \eta_{x x x}+\eta_{t}=0, \\
(c+\alpha u)\left(\xi_{x}^{2}-\xi_{t}^{1}\right)-\xi_{t}^{2}+\beta \xi_{x x x}^{2}-\alpha \eta-3 \beta \eta_{x x u}=0,
\end{gathered}
$$

where the subscripts denote partial differentiation with respect to the indicated variables, and a "prime" represents total derivative with respect to $t$.

The manipulation of (7) leads to the coefficients of Lie point symmetry generator given by

$$
\begin{gathered}
\xi^{1}=k_{1} t+k_{2}, \\
\xi^{2}=\frac{1}{3} k_{1} x+a(t), \\
\eta=\frac{1}{3 \alpha}\left[3 a^{\prime}(t)-2 k_{1}(c+\alpha u)\right],
\end{gathered}
$$

where $k_{1}, k_{2}$ are arbitrary constants, and $a(t)$ is an arbitrary function which satisfies the classifying relation

$$
5 \alpha k_{1} F(t)+3 \alpha\left(k_{1} t+k_{2}\right) F^{\prime}(t)=3 a^{\prime \prime}(t) .
$$

Assume that $F(t)$ is an arbitrary smooth function of $t$; then from the classifying (9) we obtain

$$
k_{1}=0, \quad k_{2}=0, \quad a(t)=k_{3} t+k_{4},
$$

where $k_{3}$ and $k_{4}$ are arbitrary constants. Thus, we have a twodimensional principal Lie algebra, which is spanned by the operators

$$
X_{1}=\partial_{x}, \quad X_{2}=\alpha t \partial_{x}+\partial_{u} .
$$

Our goal is to obtain the functional forms of the forcing term, $F(t)$, for which the principal Lie algebra is extended. Therefore, the analysis of the classifying relation (9) considering the cases $a^{\prime \prime}(t)=0$ and $a^{\prime \prime}(t) \neq 0$ yields the various forms of $F(t)$, and their corresponding extensions of the principal Lie algebra are given in Table 1 . It is worth mentioning that some of the obtained symmetry classification results are comparable with those found in $[4,5]$. It is, however, noted that in [6] a more general case is considered. 
TABLE 2: Table of commutators.

\begin{tabular}{lccc}
\hline$\left[X_{i}, X_{j}\right]$ & $X_{1}$ & $X_{2}$ & $X_{3}$ \\
\hline$X_{1}$ & 0 & 0 & $\alpha X_{1}$ \\
$X_{2}$ & 0 & 0 & $-3 n \alpha^{2} X_{1}-2 \alpha X_{2}$ \\
$X_{3}$ & $-\alpha X_{1}$ & $3 n \alpha^{2} X_{1}+2 \alpha X_{2}$ & 0 \\
\hline
\end{tabular}

Here $\left[X_{i}, X_{j}\right]=X_{i}\left(X_{j}\right)-X_{j}\left(X_{i}\right) ; i, j=1,2,3$ is the commutator operation.

\section{Symmetry Reductions and Exact Solutions}

It can be seen from Table 1 that the symmetry Lie algebra is three-dimensional for the first three cases and fourdimensional in the last case. We consider Case 3 to illustrate the procedure involved in performing similarity reductions. Since the symmetry Lie algebra is three-dimensional, we look for solutions invariant under the linear combination of the operators

$$
\begin{gathered}
X_{1}=\partial_{x}, \quad X_{2}=\alpha t \partial_{x}+\partial_{u}, \\
X_{3}=3 \alpha(n+t) \partial_{t}+\alpha x \partial_{x}-2(c+\alpha u) \partial_{u},
\end{gathered}
$$

which are the symmetries of the equation

$$
u_{t}+c u_{x}+\alpha u u_{x}+\beta u_{x x x}=F_{0}(n+t)^{-5 / 3} \text {. }
$$

In order to obtain all the possible invariant solutions, the most systematic procedure is to determine the optimal system of one-dimensional subalgebras $[10,11]$ for $(13)$. We follow the approach given in [10] by firstly computing the commutators of the symmetry Lie algebra (12) and thereafter obtaining the adjoint representations (the calculations are summarized in Tables 2 and 3, resp.).

We use Table 3 to simplify the linear combination of operators (12) given by

$$
\Gamma=a_{1} X_{1}+a_{2} X_{2}+a_{3} X_{3}
$$

for some constants $a_{1}, a_{2}$, and $a_{3}$.

Firstly, we let $a_{3} \neq 0$ (take $a_{3}=1$ ). The operator (14) becomes

$$
\Gamma=a_{1} X_{1}+a_{2} X_{2}+X_{3}
$$

We eliminate $a_{2} X_{2}$ by acting $\operatorname{Ad}\left(e^{a_{2} X_{2}}\right)$ on (15) and obtain

$$
\Gamma^{\prime}=a_{1}^{\prime} X_{1}+X_{3}
$$

for some constant $a_{1}^{\prime}$. Likewise, in order to eliminate $a_{1}^{\prime} X_{1}$, we act on (16) by $\operatorname{Ad}\left(e^{\left(a_{1}^{\prime} / \alpha\right) X_{1}}\right)$ to get $\Gamma^{\prime \prime}=X_{3}$.

Next we let $a_{3}=0\left(a_{2} \neq 0\right)$. We take $a_{2}=1$, and from (14) we have

$$
\Gamma=a_{1} X_{1}+X_{2}
$$

If $a_{1} \neq 0$ (i.e., $a_{1}>0$ or $a_{1}<0$ ), then we obtain $\Gamma=\lambda X_{1}+X_{2}$, where $\lambda= \pm 1$.

Finally, we let $a_{2}=a_{3}=0$. The operator (14) reduces to $\Gamma=X_{1}$ for $a_{1}=1$.
Therefore, an optimal system of one-dimensional subalgebras is given by $\left\{X_{1}, X_{2}, \lambda X_{1}+X_{2}, X_{3}\right\}$.

Next we utilize the optimal system to construct the invariant solutions of (13). However, the invariance under space translation, that is, $X_{1}=\partial_{x}$, is trivial hence, it is not considered. The other cases are presented as follows.

Case 1. Invariance under $X_{2}$ : the corresponding characteristic system is given by

$$
\frac{d t}{0}=\frac{d x}{\alpha t}=\frac{d u}{1}
$$

the solution of which leads to the invariants

$$
C_{1}=t, \quad C_{2}=u-\frac{x}{\alpha t} .
$$

Therefore, the invariant solution takes the form

$$
u(t, x)=f(t)+\frac{x}{\alpha t},
$$

where $f(t)$ satisfies the reduced equation

$$
\frac{c}{\alpha t}-\frac{F_{0}}{(n+t)^{5 / 3}}+\frac{f}{t}+\frac{d f}{d t}=0 .
$$

Now solving (21) for $f(t)$ and substituting into (20), we obtain the exact solution

$$
u(t, x)=\frac{1}{t}\left[f_{0}+F_{0}\left\{3+\frac{3 n}{2(n+t)}\right\}(n+t)^{1 / 3}+\frac{x-c}{\alpha}\right],
$$

where $f_{0}$ is an arbitrary constant.

Case 2. The invariance under $\lambda X_{1}+X_{2}(\lambda \neq 0)$ yields the invariant solution

$$
u(t, x)=f(t)-\frac{x}{\alpha t+\lambda},
$$

where $f(t)$ is an arbitrary smooth function of its argument. Upon substitution of (23) into (13) and solving the resulting ordinary differential equation (ODE), we have

$$
f(t)=\frac{1}{\alpha t+\lambda}\left\{f_{0}-c t+\frac{3 F_{0}(\lambda-3 n \alpha-2 t \alpha)}{2(n+t)^{2 / 3}}\right\},
$$

for an arbitrary contant $f_{0}$. Therefore, we obtain the exact solution

$$
u(t, x)=\frac{1}{\alpha t+\lambda}\left\{f_{0}-c t-x+\frac{3 F_{0}(\lambda-3 n \alpha-2 t \alpha)}{2(n+t)^{2 / 3}}\right\} .
$$

Case 3. Invariance under $X_{3}$ : in this case, the invariant solution assumes the form

$$
u(t, x)=\frac{f(z)}{(n+t)^{2 / 3}}-\frac{c}{\alpha},
$$

where $z=x(n+t)^{-1 / 3}$ is the similarity variable. The function $f(z)$ is an arbitrary function which satisfies the third-order ODE

$$
3 \beta \frac{d^{3} f}{d z^{3}}+\left(3 \alpha \frac{d f}{d z}+2\right) f+z \frac{d f}{d z}-3 F_{0}=0 .
$$


TABLE 3: Table of adjoint representations.

\begin{tabular}{lccc}
\hline $\operatorname{Ad}\left(e^{\epsilon X_{i}}\right) X_{j}$ & $X_{1}$ & $X_{2}$ & $X_{3}$ \\
\hline$X_{1}$ & $X_{1}$ & $X_{2}$ & $X_{3}-\alpha \epsilon X_{1}$ \\
$X_{2}$ & $X_{1}$ & $X_{2}$ & $X_{3}+\epsilon\left(3 n \alpha^{2} X_{1}+2 \alpha X_{2}\right)$ \\
$X_{3}$ & $e^{\epsilon \alpha} X_{1}$ & $e^{-2 \alpha \epsilon} X_{2}+3 n \alpha\left(e^{-\epsilon \alpha}-1\right) X_{1}$ & $X_{3}$ \\
\hline
\end{tabular}

Here $\operatorname{Ad}\left(e^{\epsilon X_{i}}\right) X_{j}=X_{j}-\epsilon\left[X_{i}, X_{j}\right]+(1 / 2 !) \epsilon^{2}\left[X_{i},\left[X_{i}, X_{j}\right]\right]-\cdots$ is the adjoint representation where $\epsilon$ is a real number.

\section{Conclusion}

In this work, the $\mathrm{KdV}$ equation with a forcing term was investigated using the Lie symmetry approach. The direct analysis of the classifying equation was employed to obtain the functional forms of the forcing term, which include power law and linear and constant time dependence. The threeand four-dimensional symmetry Lie algebras were obtained, respectively, for these forms of the forcing term. The optimal system of one-dimensional subalgebras of the Lie algebra of the invariant equation with the forcing term having the power law nonlinearity was obtained. As a result, for the same invariant equation, exact solutions were derived and the symmetry reduction was performed in the case where exact solutions were not obtained.

\section{Acknowledgment}

M. Molati thanks the North-West University, Mafikeng Campus, for the Postdoctoral Fellowship.

\section{References}

[1] D. J. Korteweg and G. de Vries, "On the change of form of long waves advancing in a rectangular canal, and on a new type of long stationary waves," Philosophical Magazine, vol. 39, no. 240, pp. 422-443, 1895.

[2] A. H. Salas, "Computing solutions to a forced KdV equation," Nonlinear Analysis: Real World Applications, vol. 12, no. 2, pp. 1314-1320, 2011.

[3] A.-M. Wazwaz, "A study on $\mathrm{KdV}$ and Gardner equations with time-dependent coefficients and forcing terms," Applied Mathematics and Computation, vol. 217, no. 5, pp. 2277-2281, 2010.

[4] G. F. Jefferson, "On the second-order approximate symmetry classification and optimal systems of subalgebras for a forced Korteweg-de Vries equation," Communications in Nonlinear Science and Numerical Simulation, 2013.

[5] M. L. Gandarias and M. S. Bruzón, "Some conservation laws for a forced KdV equation," Nonlinear Analysis: Real World Applications, vol. 13, no. 6, pp. 2692-2700, 2012.

[6] V. Listopadova, O. Magda, and V. Pobyzh, "How to find solutions, Lie symmetries, and conservation laws of forced Korteweg-de Vries equations in optimal way," Nonlinear Analysis: Real World Applications, vol. 14, no. 1, pp. 202-205, 2013.

[7] A. G. Johnpillai and C. M. Khalique, "Group analysis of KdV equation with time dependent coefficients," Applied Mathematics and Computation, vol. 216, no. 12, pp. 3761-3771, 2010.

[8] M. Molati and M. P. Ramollo, "Symmetry classification of the Gardner equation with time-dependent coefficients arising in stratified fluids," Communications in Nonlinear Science and Numerical Simulation, vol. 17, no. 4, pp. 1542-1548, 2012.

[9] G. W. Bluman and S. Kumei, Symmetries and Differential Equations, vol. 81, Springer, New York, NY, USA, 1989.

[10] P. J. Olver, Applications of Lie Groups to Differential Equations, vol. 107, Springer, New York, NY, USA, 1986.

[11] L. V. Ovsiannikov, Group Analysis of Differential Equations, Academic Press, New York, NY, USA, 1982. 


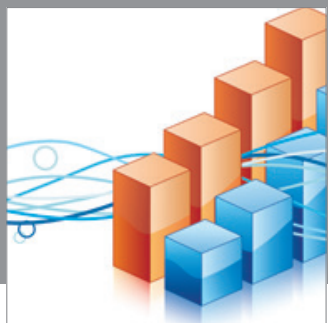

Advances in

Operations Research

mansans

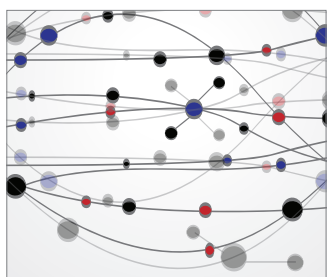

The Scientific World Journal
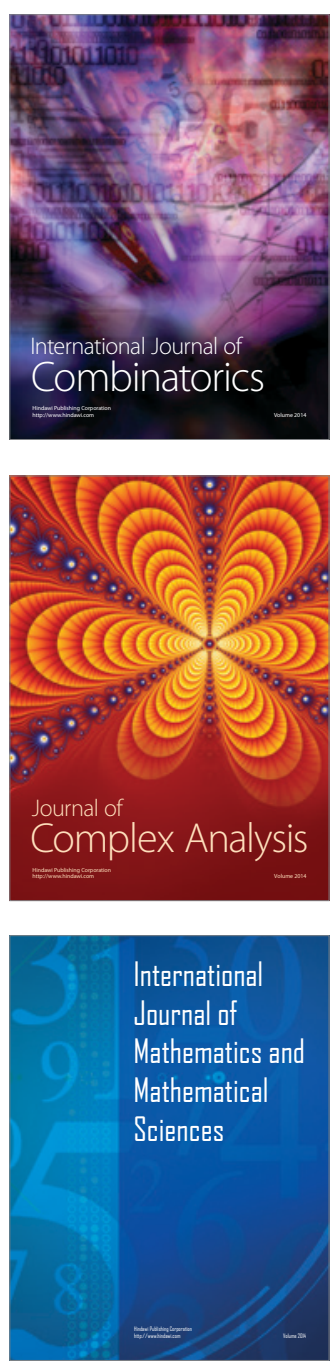
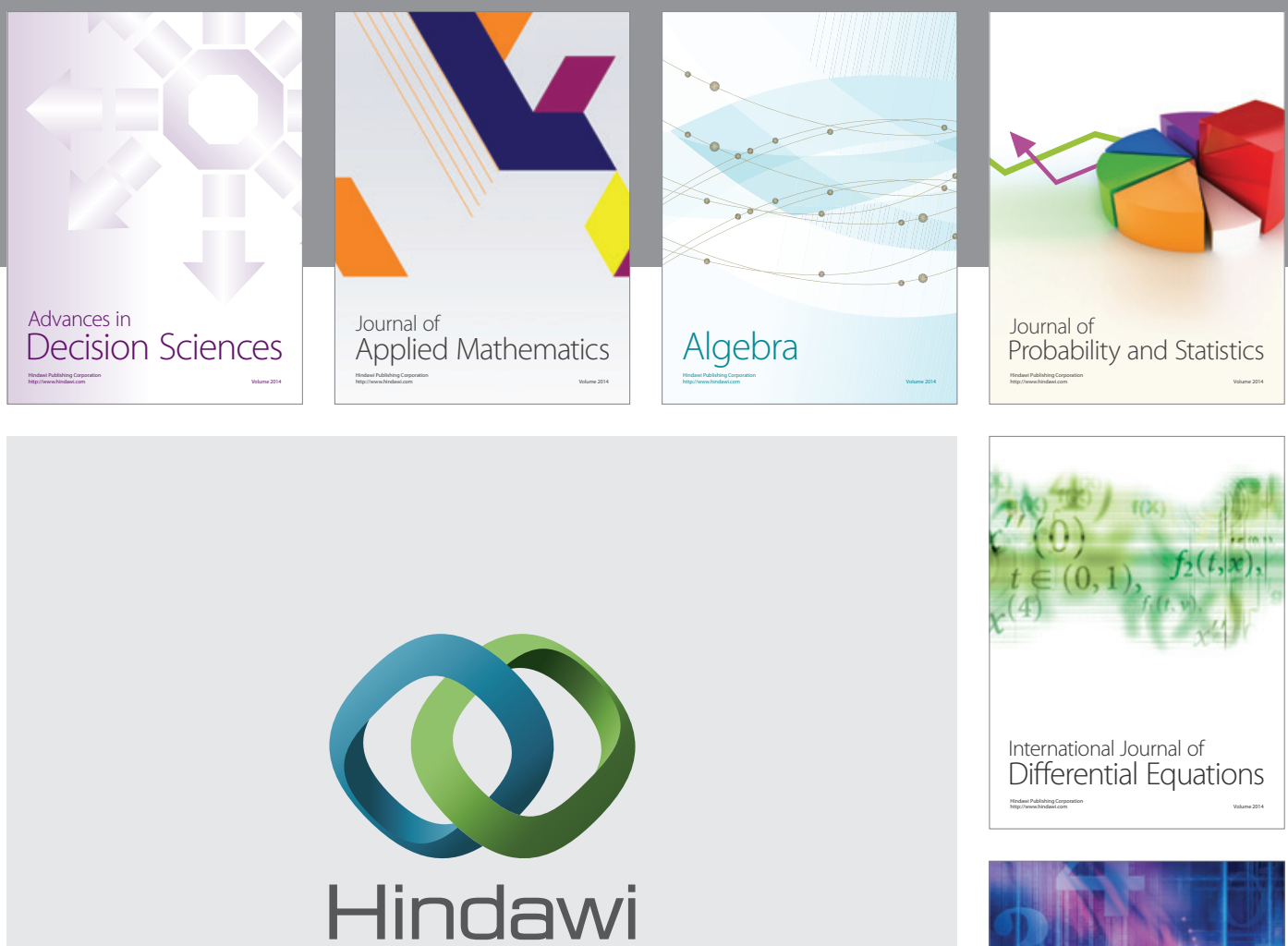

Submit your manuscripts at http://www.hindawi.com
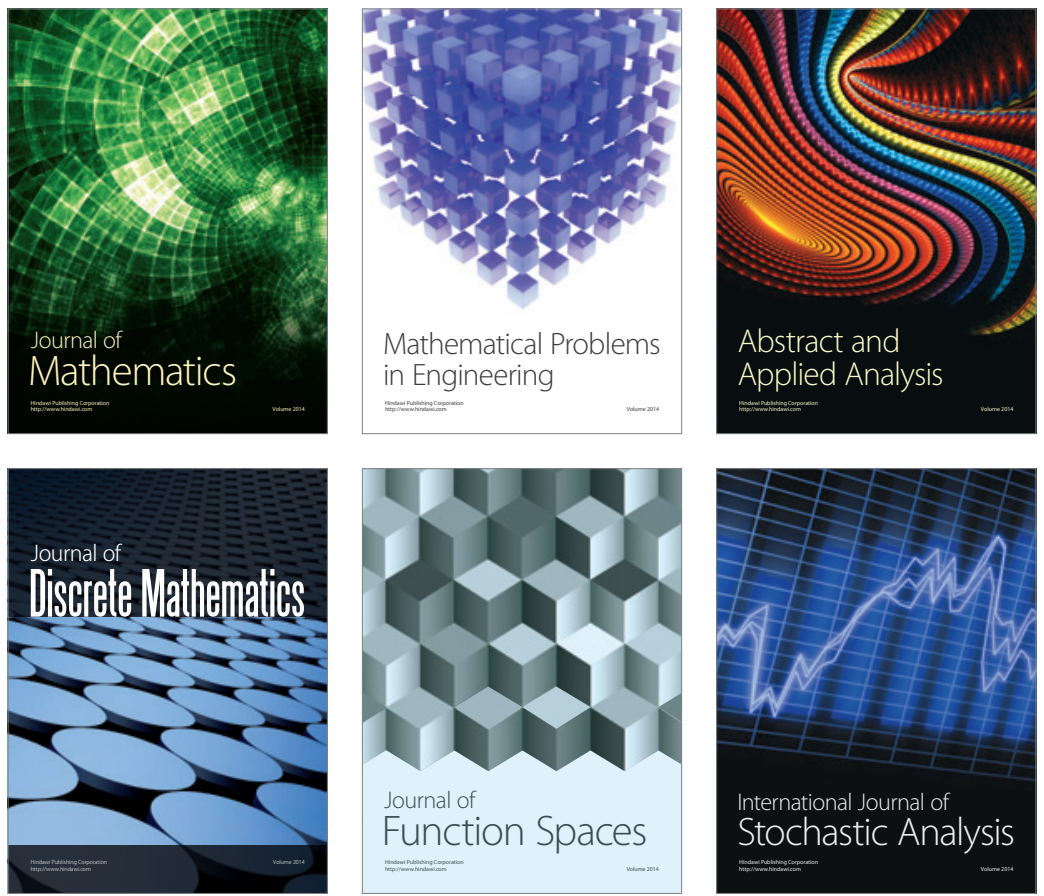

Journal of

Function Spaces

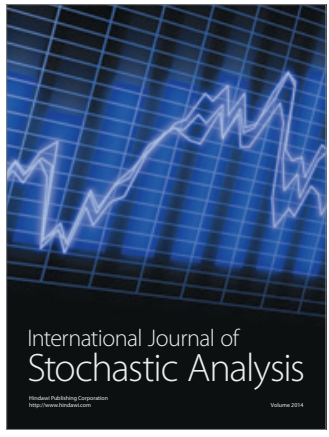

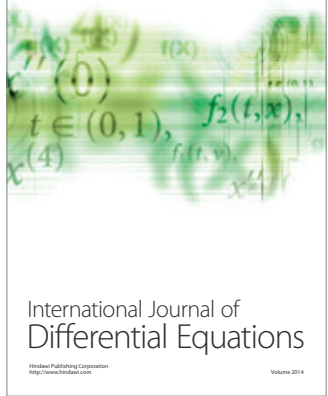
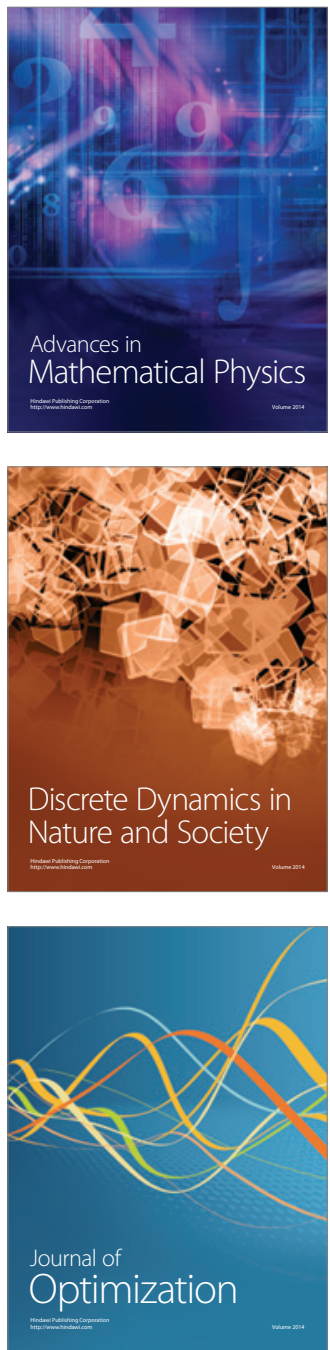